\title{
LA ÉTICA COMO MEDIO PARA LA VIDA BUENA: UNA REFLEXIÓN DESDE A. MACINTYRE
}

\author{
Ethics as a way for the Good Life: A reflection from A. MacIntyre
}

\author{
David Lorenzo Izquierdo \\ Centro Universitario San Rafael-Nebrija \\ (Universidad Antonio de Nebrija)
}

Sumário: En la actualidad, la ética está excesivamente centrada en la reflexión y en el establecimiento de reglas, de normas. Muchos debates sobre ética empiezan o acaban en la reflexión sobre qué normas o reglas deben aceptarse pública o universalmente, sobre cuáles de ellas deben ser leyes civiles, sobre qué comportamientos la ley civil debería prohibir y por qué. A juicio del autor, este hecho supone un reduccionismo y hace olvidar que lo más importante de la ética es el desarrollo integral del individuo. Las reglas tienen su importancia en ética, pero no son el factor más relevante en ella porque no son suficientes para ayudar al individuo al desarrollo de sus capacidades, para su vida buena. El artículo muestra cómo la virtud juega un papel fundamental en ese proceso.

Palabras clave. Maclntyre, ética, virtud, regla/ley, felicidad.

\begin{abstract}
Contemporary ethics is excessively focused on thinking and setting rules and norms. Many debates on ethics begin or finish in the reflection about which norms and rules should be publically or universally accepted, about which one should be civil laws, about which behaviors the civil law should prohibit and why. In our view, this fact presupposes a reductionism and makes us to forget that the most important task of ethics is to provide the full flourishing of individual. The norms have an important role in ethics, but they are not its most relevant factor because they are not sufficient to help individuals to develop their capacities for the good life. This paper shows how virtue plays a fundamental role in that process.
\end{abstract}

Key words: Maclntyre, Ethics, virtue, rule/law, happiness.

\section{Introducción}

El pensamiento de Alasdair MacIntyre es muy rico en temas y en los modos de abordarlos. Su vasta formación y sus amplios intereses han hecho posible que el autor escriba sobre temas de diversos campos y, a la vez, que su obra haya sido de interés en 
diversas disciplinas humanísticas: ética, sociología, antropología, teología, filosofía política, etc. No obstante, el ámbito en el que su figura e influencia son más importantes es la ética.

En este trabajo, me gustaría tratar un tema del ámbito de la ética, un tema que es de especial interés y preocupación para mí y también —creo- para Maclntyre, aunque el autor no lo aborde de manera sistemática en ninguno de sus trabajos. El tema es la consideración de la ética como medio de mejora personal para el individuo. Es decir, la consideración de la ética esencialmente como medio para la vida buena, para el desarrollo de las capacidades del individuo.

Hablar de la ética en esos términos no es, por supuesto, algo nuevo, y, de alguna manera, puede resultar algo evidente. La ética, como disciplina de conocimiento, se centra en el análisis del comportamiento humano desde el punto de vista de su corrección/incorrección (o bondad/maldad). Muchas ciencias estudian el comportamiento humano, pero la ética lo hace desde su propio punto de vista y abordaje (juzgando su corrección o incorrección).

No obstante, se puede decir que esa reflexión sobre el comportamiento humano se dirige, en último término, a orientarlo, a mejorarlo. Esta tesis, que puede resultar evidente, recibe en la actualidad —creo- poca atención dentro del ámbito de la ética. En las aulas universitarias, en los congresos de filosofía, en discusiones públicas, etc., muchos debates sobre ética empiezan o acaban en la reflexión sobre qué normas o reglas deben aceptarse pública o universalmente, sobre cuáles de ellas deben ser leyes civiles, sobre qué comportamientos la ley civil debería prohibir y por qué.

El interés y la reflexión sobre las reglas o las normas es una parte fundamental de la ética, por supuesto, pero no es - a mi juicio- el punto central de esta disciplina. En la actualidad parece que la atención a las normas - a la vertiente normativa de la ética - tiene un papel preeminente, y parece que la ética tenga como fin fundamental el establecimiento de normas morales y civiles para regular (o limitar en muchos casos) 
el comportamiento humano. Por supuesto, la reflexión y el establecimiento de normas es una parte de la ética, pero no es la única y - lo que es más importante - ni la principal, pues la ética se dirige, en último término, a la guía de la vida del individuo, al desarrollo de sus capacidades, en definitiva: a la vida buena. Y ésta no se consigue sólo a través de normas, sino a través de un entramado de conceptos interrelacionados (virtud, bien, etc.).

\section{1. Ética y vida humana}

La primera idea importante para la consideración de la ética que se propone aquí es que la ética forma parte esencial de la vida. Esta idea puede resultar evidente, pero lo importante en ella es la característica esencial: la ética no sólo forma parte de la vida sino que juega un papel esencial en ella. Y ese papel lo juega porque la ética no es sólo una reflexión teórica del comportamiento sino, sobre todo, una actividad práctica. El bien hecho - practicado- es a lo que se dirige la ética, no -o no sólo- al bien pensado - el bien como objeto de especulación-.

Afirma Maclntyre que ambas vertientes del bien (la especulativa o teórica y la práctica) están íntimamente unidas. Para el autor, la relación entre la filosofía y la vida personal es fundamental, aspecto importante para todo pensamiento de corte aristotélico: las preguntas morales de la persona - afirmaba en su artículo "Plain Persons and Moral Philosophy: Rules, Virtues and Goods" - exigen reflexión filosófica, y la reflexión moral de la filosofía no es completa si no se puede encarnar en vidas de personas concretas (Maclntyre, 1992: 136-137). Prueba de ello es que nuestro autor dedica uno de sus últimos libros publicados, Edith Stein: A Philosophical Prologue (2006a), a ver la conexión de la vida, las circunstancias y el carácter personal de Edith Stein con la configuración inicial de su pensamiento.

Maclntyre muestra la importancia de la vertiente práctica de la ética al explicar la estructura básica de la ética según Aristóteles. El autor basa esa estructura en tres puntos (Maclntyre, 1981: 52-53): 
1) la naturaleza humana tal como es,

2) la naturaleza humana que ha de ser, como ha de ser (telos) según su esencia,

3) el camino entre ambos puntos: la ética.

A la luz de este esquema, puede entenderse por qué la ética es tan esencial para la vida. La ética no es sino el camino hacia el telos propio del ser humano, telos que depende de las cualidades o propiedades esenciales del ser humano.

La ética es, pues, una realidad mediatizada: es un medio para un fin, para el telos propio del ser humano (y del individuo). Sin embargo, la ética no es un mero medio externo con respecto a dicho telos. Maclntyre se sirve en varias ocasiones (hablando del concepto de virtud) de la diferencia entre un medio interno y un medio externo (1981: 184; 1988: 131-132). Un medio externo es un medio distinto -e independiente ontológicamente- del fin al que se dirige, con el que guarda una relación extrínseca: es el caso de un martillo y el clavo. El martillo es un medio (externo) para clavar el clavo. Un medio interno, por el contrario, guarda una relación intrínseca con el fin al que se dirige: es el caso de una jugada de ajedrez y la partida de la que forma parte (este ejemplo es de Maclntyre). La ética, por tanto, es un medio, pero un medio interno con respecto al telos último del ser humano. El ser de la ética no es independiente de ese telos, ni el ser de éste lo es de aquélla.

Ese telos es el fin último del ser humano, su bien máximo. Es el concepto aristotélico de eudaimonia, que Maclntyre asume con el término florecimiento (flourishing). El concepto de florecimiento (flourishing) es desarrollado por nuestro autor en Dependent Rational Animals (1999). En esa obra, afirma que lo que necesita un miembro de una especie para florecer es desarrollar las facultades características ("distinctive powers") que posee qua miembro de esa especie (Maclntyre, 1999: 6364). Florecer, por tanto, significa desarrollar las facultades propias de un individuo como hombre (en este caso); o, en otras palabras, cómo tendría que vivir el ser humano (Maclntyre, 1999: 76-77). De hecho, afirma Russell que ésta es la cuestión 
que, de un modo u otro, ha guiado siempre la reflexión de Maclntyre: cómo vivir, qué vida es mejor, qué hace bueno a alguien (Russell, 2003: iv).

Cada ser humano, pues, tiende a su florecimiento, que es su telos último, y la ética es el medio interno -el camino- para alcanzarlo. Maclntyre comparte (de fondo) la concepción aristotélico-tomista del bien, según la cual el bien vendría a ser aquello que perfecciona o conviene a una naturaleza (Tomás de Aquino, Suma de teología, I-II, q. 54, a. 3, ad 2), y, por tanto, su fin, aquello a lo que tiende (Tomás de Aquino, Suma de teología, I-II, q. 1, a. 4; Aristóteles, Metafísica I, 983a, 32; Ética nicomáquea, I, 1094a, 2-3; X, 1173a, 1). Ese fin último es el que orienta los fines inmediatos del individuo, es decir, los bienes que va eligiendo.

Maclntyre habla también del concepto de bien con respecto a los papeles sociales que todo individuo desempeña. Sostiene el autor que, en toda sociedad, los individuos, por sus preferencias, situación, grupo, etc., representan o encarnan papeles (roles): dentista, pensionista, sacerdote, etc., papeles que tienen su telos propio (Maclntyre, 1981: 28-29, 219-220). En este sentido, una acción es buena en función de una actividad o de una práctica socialmente establecida, y se juzga como bueno a un agente y a su acción en función de los bienes intrínsecos de ese papel. En este ámbito, una persona es buena como madre, como pescador, agricultor, soldado, etc. ${ }^{1}$. (MacIntyre, 1992: 151; 1999: 66).

\section{Florecimiento, unidad narrativa y virtud}

Hasta ahora, hemos visto cómo la ética supone el camino (el medio interno) entre la naturaleza humana y su telos (la naturaleza como ha de ser). Hay, pues, una relación entre esos dos puntos: la naturaleza humana como es y la naturaleza humana como ha de ser. Esa relación es lo que permite a Maclntyre - en mi opinión- concebir

\footnotetext{
${ }^{1}$ Señala MacIntyre en Against the Self-Images of the Age que, contra la separación hecho-valor, Marx sostuvo que hablar del papel social -clase- de una persona implica hablar de lo que debe hacer: los valores son dados, no elegidos, y no son independientes de los hechos: separarlos es ya una valoración (Maclntyre, 1971: 92).
} 
la vida como una unidad narrativa, término acuñado y desarrollado en After Virtue (Maclntyre, 1981: 118, 130).

Partiendo de la reflexión de nuestro autor, se puede afirmar que la vida humana es una unidad porque puede concebirse como un todo, cuyas partes están interrelacionadas en función del fin (el florecimiento) ${ }^{2}$ : la vida es unidad porque su estructura es teleológica. Y esa unidad es narrativa porque ese fin unitario hace de la vida algo con un inicio, un desarrollo y un final; y porque tal fin se define, se comprende y se práctica en una historia concreta, en un despliegue y una actividad: no se da acabadamente de una vez sino que se va dando, no es algo estático sino dinámico; no se define: se re-define. Por ello, la muerte debe entenderse como punto en el que se emite un juicio sobre el logro del bien que fundamentaba y orientaba la vida (MacIntyre, 1990a: 196-197).

El individuo, por tanto, a medida que va viviendo, va desarrollando y aplicando su propia concepción del bien, del telos. Es decir, va descubriendo el florecimiento y los bienes que lo conforman. En este sentido, conviene señalar lo que indicaba Aristóteles en su Ética nicomáquea (VII, 1152b, 25-30): que el bien tiene un sentido absoluto y un sentido individual. Hay bienes que son tales para todo ser humano por el hecho de serlo, y otros que son sólo bienes para el individuo (en función de su carácter y circunstancias personales).

Maclntyre procura siempre orientar su pensamiento al individuo concreto. Recurre, por supuesto, a generalizaciones, pero siempre teniendo como telón de fondo que la vida humana es vida primariamente individual, que cada florecimiento es un florecimiento individual. Por tanto, cada individuo construye o elabora su propia narración del florecimiento. Maclntyre pretende reflexionar sobre este hombre más que sobre el hombre en general. Es el individuo, con sus características y circunstancias propias, el que florece.

\footnotetext{
${ }^{2}$ Maclntyre no relaciona los conceptos de unidad narrativa y florecimiento aun cuando, a mi juicio, se complementan - más aun: se requieren - estrechamente.
} 
El medio fundamental de ese florecimiento, lo que permite al ser humano florecer, o más bien aquello en lo que consiste el florecimiento, es la virtud. Maclntyre hereda de Aristóteles la importancia de la virtud como factor central del desarrollo moral del individuo. Muchas de sus obras incluyen, directa e indirectamente, una reflexión sobre el concepto de virtud.

Nuestro autor aborda la virtud en relación con el florecimiento pero, primariamente, en relación con la práctica. Según Maclntyre, la vida humana (las relaciones, el desarrollo individual, etc.) tiene como marco natural las prácticas. En After Virtue, el autor define práctica como una actividad cooperativa socialmente establecida, actividad con unas reglas intrínsecas, con un bien interno propio (su perfección, su finalidad) y con unos bienes externos (esos bienes complementarios a la práctica: dinero, prestigio, etc.). Desde el punto de vista de las prácticas, una persona es buena como padre, como pintor, como músico o constructor de barcos, etc., lo cual requiere ciertas virtudes por parte del sujeto. Las acciones humanas tienen lugar siempre en unas prácticas, es decir, en actividades supeditadas a un bien interno (el telos propio de cada práctica), a unas reglas, a unos modelos, a ciertas virtudes y a cierta historia (Maclntyre, 1981: 187-194; 1990a: 64-65; 2009: 12).

En función de los conceptos de florecimiento (unidad narrativa) y de práctica, MacIntyre va construyendo o acotando la definición de virtud en After Virtue y Dependent Rational Animals ${ }^{3}$. Las virtudes no sólo ayudan a las prácticas sino también a la constancia en la búsqueda de lo bueno y al conocimiento de ello y del propio individuo: "[Las virtudes son] aquellas disposiciones que no sólo mantienen las prácticas y nos permiten alcanzar los bienes internos a las prácticas, sino que nos

\footnotetext{
${ }^{3}$ La primera definición de virtud - parcial y provisional- que ofrece en After Virtue se refiere más a la práctica y dice: "Una virtud es una cualidad humana adquirida, cuya posesión y ejercicio tiende a hacernos capaces de lograr aquellos bienes que son internos a las prácticas y cuya carencia nos impide efectivamente el lograr cualquiera de tales bienes" ("A virtue is an acquired human quality the possession and exercise of which tends to enable us to achieve those goods which are internal to practices and the lack of which effectively prevents us from achieving any such goods") (Maclntyre, 1981: 191). Para un análisis completo del concepto de virtud que Maclntyre expone en After Virtue, Whose Justice? y Three Rival Versions: MAURI, Liberalisme i llibertat ("Alasdair Maclntyre: la tradició de les virtuts"), Qüestions de vida cristiana, n. 194, Publicacions de l'Abadia de Montserrat.
} 
sostendrán también en el tipo pertinente de búsqueda de lo bueno, ayudándonos a vencer los riesgos, peligros, tentaciones y distracciones que encontremos y procurándonos creciente autoconocimiento y creciente conocimiento del bien" ${ }^{\prime 4}$ (Maclntyre, 1981: 219). Complementariamente, Maclntyre afirma, en Dependent Rational Animals, que las virtudes son excelencias (excellences), son "...las cualidades intelectuales y de carácter que permiten a una persona identificar los bienes pertinentes y emplear las habilidades necesarias para conseguirlos..." ${ }^{5}$ (Maclntyre, 1999: 91-92).

Las virtudes se manifiestan e influyen en todos los ámbitos de la vida del individuo. Si bien nacen o se dan inicialmente en las prácticas, su alcance es mucho mayor: la vida como unidad (con su telos) y el razonamiento práctico (Maclntyre, 1981: 201-203; 1999: 120). A lo largo del tratamiento de distintos temas (práctica, florecimiento, etc.), el autor va poniendo ejemplos de distintos momentos o esferas del ser humano en que las virtudes aparecen como necesarias. Pero, finalmente, viene a decir en síntesis que las virtudes influyen en la actitud o reacción del individuo en distintas situaciones, en las relaciones sociales (prácticas, educación, etc.) y en el razonamiento práctico de todo agente (Maclntyre, 1999: 92, 97-98, 119-120), es decir, en todos los ámbitos de la vida.

\section{Virtud y desarrollo moral del individuo}

Maclntyre propone un concepto de virtud de base aristótelico-tomista, aunque su desarrollo no parta de la metafísica (sino más bien de la práctica) ${ }^{6}$. Según Tomás de Aquino, la virtud perfecciona una facultad y su operación. El motivo es que

\footnotetext{
4 "The virtues therefore are to be understood as those dispositions which will not only sustain practices and enable us to achieve the goods internal to practices, but which will also sustain us in the relevant kind of quest for good, by enabling us to overcome the harms, dangers, temptations and distractions which we encounter, and which will furnish us with increasing self-knowledge and increasing knowledge of good".

5 "...those qualities of mind and character that enable someone both to recognize the relevant goods and to use the relevant skills in achieving them".

6 Cabe indicar, no obstante, que nuestro autor, en los últimos 15-20 años, ha ido incluyendo la metafísica en su sistema de pensamiento (que no es de tipo metafísico).
} 
perfección se dice con respecto a un fin, y el fin de la facultad es el acto, de modo que una facultad es perfecta en cuanto que se determina hacia su acto. Las virtudes, pues, son hábitos determinadores (si se me permite usar la expresión) (Suma de teología, I-II, q. 55, a. 1; I-II, q. 56, a. 6). Al implicar la perfección de una facultad y ya que un mal implica un defecto, la virtud es un hábito operativo bueno y productor de buenas obras (Suma de teología, I-II, q. 55, a. 3). Cada virtud - dice Aristóteles- es perfección de su capacidad respectiva (Ética nicomáquea, II, 1106a, 15-20).

Por eso dice Tomás de Aquino que un hábito es llamado virtud por dos razones: porque confiere aptitud a una facultad para su operación, y porque confiere al sujeto el correcto uso de una facultad (Suma de teología, I-II, q. 57, a. 1). Y dice Aristóteles que el bien del hombre es la virtud, que es la excelencia de las facultades. Una actuación u obra con excelencia es una virtud (Ética nicomáquea, I, 1098a, 9-11, 15). El sentido del término griego areté me parece el más adecuado para reflejar el contenido del término virtud.

La areté aristotélica es excelencia, es decir, la actualización mejor de una facultad. La actualización de esa facultad en acciones concretas implica que dichas acciones deben ser excelentes, es decir, lo mejor posibles (en función del sujeto, las circunstancias, etc.). Y, gracias a ellas, la propia facultad se hace excelente, esto es, mejor con respecto a su naturaleza (en un sujeto agente concreto). Se entiende así que Tomás de Aquino diga que "el acto de virtud no es más que el buen uso del libre arbitrio" ("bonus usus liberi arbitrii") (Suma de teología, I-II, q. 55, a. 1, ad 2).

Me interesa aquí destacar que, más allá de los tipos y de las clasificaciones de las distintas virtudes, lo principal es que la virtud es excelencia. Desde este sentido, se puede decir que la areté humana consiste en buscar la areté en sus acciones concretas, de modo que, por su carácter de praxis, la areté de o en dichas acciones redunde en la areté de la persona. La areté es el telos de la unidad narrativa, es decir, es la excelencia de la persona. 
Como excelencia de la persona en su globalidad, la virtud, para Aristóteles, no sólo implica capacidad de acción sino, primariamente, un modo de ser (Ética nicomáquea, I, 1098a, 15; VI, 1145a, 3-6). La virtud, en su sentido más profundo, supone una transformación del sujeto y de sus facultades. Gracias a eso - afirma Maclntyre - la virtud permite al individuo distinguir, valorar, entender y realizar los bienes de las prácticas y el florecimiento en general (Maclntyre, 1981: 198-199; 1991: 262; 1999: 112).

Conviene destacar que la virtud no sólo capacita al individuo para hacer sino, sobre todo, para ser. Y esta influencia en su ser le hace capaz de valorar correctamente (los bienes, la realidad). Es decir, según Maclntyre, la virtud no ayuda a entender un bien -aunque también - sino a valorarlo, es decir, a distinguirlo y a saber darle el valor adecuado con respecto a otros bienes y en referencia al florecimiento (bien máximo). Pienso que este aspecto de la virtud es de una importancia capital para el desarrollo moral del individuo, tema en el que se centra este artículo dentro del marco de la ética. Según Aristóteles, la virtud permite al individuo disfrutar con lo debido con lo bueno- y no disfrutar con lo indebido - con lo malo-, para lo cual el alma requiere el cultivo de hábitos (Ética nicomáquea, IX, 1172a, 22-24; X, 1179b, 26). A la vez, ese cultivo facilita al individuo el conocimiento de los principios morales (Ética nicomáquea, VII, 1151a, 10-15).

Otra de las funciones importantes de la virtud para Maclntyre (como para Aristóteles y Tomás de Aquino) es que ésta ayuda al individuo a tratar con las reglas, con las normas morales (y también civiles). Toda práctica (como la arquitectura, por ejemplo) tiene unas normas (técnicas y morales). El individuo debe asumirlas y seguirlas si quiere alcanzar el bien interno de esa práctica, bien que les da sentido. De igual manera, y como no toda práctica por el hecho de serlo es buena, existen unas normas del florecimiento. Estas normas son las que componen la ley natural y su respeto permite al individuo alcanzar el florecimiento como ser humano. Así como el florecimiento es el criterio de las prácticas, la ley natural es el criterio de las normas de 
las prácticas. Las normas, pues, tienen sentido sólo como guías hacia un bien (Maclntyre, 1990a: 133-134, 139; 1990c: 344; 1992: 143; 1994: 173; 1996: 676-677; 1999: ix-x; 2000: 113; 2006b: vii; 2009: 3, 23, 24). La virtud capacita al individuo para saber cómo aplicar una norma y, sobre todo, para poder hacerlo (aplicarla).

\section{El papel de la prudencia}

Aunque Maclntyre destaca diversas virtudes, tanto en el nivel teórico (definición del bien y distinción de bienes particulares) como en el práctico (aplicación/realización efectiva de un bien), destaca especialmente el papel de una virtud: la prudencia, la phronesis. La prudencia es la virtud central de la vida práctica para Aristóteles y Tomás de Aquino (Maclntyre, 1990b: 41-42). Nuestro autor define la phronesis como "...el ejercicio de la capacidad de aplicar verdades acerca del bien para tal o cual tipo de persona o para personas en general y en ciertos tipos de situaciones a uno mismo en ocasiones particulares. El 'phronimos' es capaz de juzgar no sólo qué verdades son relevantes para él en su situación particular sino que también, a partir de ese juicio y de su percepción de los aspectos relevantes de sí mismo y de su situación, cómo actuar rectamente"17 (Maclntyre, 1988: 115-116). También habla de ella como "...la virtud consistente en ser capaz de remitirse, en las situaciones particulares, a los universales pertinentes y actuar de modo que el universal se encarne en el particular"8 (Maclntyre, 1990a: 139).

No obstante, la prudencia no es autosuficiente: requiere las virtudes morales para su formación y crecimiento. De hecho, como para Aristóteles y Tomás de Aquino, la relación entre las virtudes morales y la phronesis es de mutua necesidad para Maclntyre. Sostiene nuestro autor que toda virtud moral "...no puede ejercerse sin que

\footnotetext{
7"...the exercise of a capacity to apply truths about what it is good for such and such a type of person or for persons as such to do generally and in certain types of situation to oneself on particular occasions. The 'phronimos' is able to judge both which truths are relevant to him in his particular situation and from that judgment and from his perception of the relevant aspects of himself and his situation to act rightly". 8 "...the virtue of being able in particular situations to bring to bear the relevant universals and to act so that the universal is embodied in the particular".
} 
se ejerza también la 'phronesis'"'9 (Maclntyre, 1988: 115-116). A la vez, la decisión de un acto concreto y su ejecución, que son guiadas por la prudencia y son el producto de la razón práctica, son imposibles de modo correcto sin las virtudes morales (del carácter): no hay racionalidad práctica sin ellas (Maclntyre, 1988: 135-136; 1998: 104). El motivo que arguye el autor es que, sin las virtudes morales, los deseos no pueden estar informados por la razón, no pueden transformarse ni ser eficaces en cuanto deseos para lo que la razón prescriba. "Son las virtudes las que capacitan al deseo, que es la 'prohairesis', para ser un deseo racional"10 (Maclntyre, 1988: 136-137).

En resumen, la importancia de la virtud de la prudencia radica para MacIntyre en dos puntos:

1) Capacita al individuo para llevar particulares bajo conceptos universales, de modo que permite distinguir circunstancias relevantes según el bien general buscado.

2) Una acción correcta concreta no es producto de aplicar reglas, aunque las requiera y las contenga: la aplicación de reglas (cómo, cuándo, cuál, etc.) no depende de una regla, sino de la prudencia ${ }^{11}$ (Maclntyre, 1990b: 41-42; 1994: 183; 1998: 106).

Hay, pues, una relación estrecha entre la prudencia y las circunstancias del individuo (personales y del entorno). Hemos dicho antes que el bien tiene dos niveles: el universal y el individual. El florecimiento del individuo se da siempre en unas circunstancias (personales, históricas, sociales, etc.) determinadas. En ellas, el individuo puede prever ciertas cosas y no otras, puede tener poder sobre algunas de

\footnotetext{
9 "...cannot be exercised without 'phronesis' also being exercised".

10 "It is the virtues which enable the desire which is 'prohairesis' to be rational desire". Maclntyre señala aquí la distinción entre orexis, deseo sentido, y prohairesis, que es el deseo guiado por la razón (1990a: 64-65).

${ }^{11}$ No obstante, como se ha comentado, el marco de aplicación general de las reglas morales es el definido por los preceptos de la ley natural (especialmente los negativos). De hecho, dice Maclntyre: "La adquisición de esa virtud [phronesis] es imposible sin reconocer la autoridad racional de los preceptos de la ley natural, sobre todo de esos preceptos negativos que no admiten excepción" ("The acquisition of that virtue [phronesis] is impossible without a recognition of the rational authority of the precepts of the natural law, most of all perhaps of the negative exceptionless precepts" -la traducción al español mía-). Por ello, tales preceptos capacitan a la racionalidad, no la merman, y son constitutivos de la autonomía humana (MacIntyre, 1994: 183).
} 
ellas y sobre otras, no. Lo expresa Maclntyre diciendo que la vida del hombre es una suma de impredecibilidad y teleología (Maclntyre, 1981: 215-216).

Dicha teleología no implica determinismo: la vida humana es -en palabras de nuestro autor - una mezcla de predecibilidad y de impredecibilidad. La predecibilidad hace posibles los compromisos y los proyectos, que son necesarios para el sentido de la vida y la identidad personal. La impredecibilidad se manifiesta en la vulnerabilidad y la fragilidad de esos proyectos, y es básica para el avance y sentido personales (MacIntyre, 1981: 103-104). "Si la vida ha de tener sentido, es necesario que podamos comprometernos en proyectos a largo plazo, y esto requiere predecibilidad; si la vida ha de tener sentido, es necesario que nos poseamos a nosotros mismos y no que seamos meras criaturas de los proyectos, intenciones y deseos de los demás, y esto requiere impredecibilidad"12 (Maclntyre, 1981: 104).

Las circunstancias, pues, que son un infinito entramado de factores predecibles e impredecibles, son el ámbito de desarrollo moral del individuo. Es ahí donde éste debe descubrir y realizar el bien que conforma su florecimiento. Nos parece importante hacer hincapié en esta característica de la existencia y del florecimiento humanos porque la corrección técnica y moral en la gestión de esas circunstancias depende principalmente del carácter del individuo, es decir, de sus virtudes, que disponen su ser y sus facultades a la acción mejor a la luz de esas circunstancias determinadas. Sólo la virtud prepara al individuo para saber responder y actuar correctamente ante la gran gama de circunstancias que rodean su razonamiento práctico. Las reglas morales, necesarias y útiles, no pueden definir (o no totalmente) la decisión correcta que debe tomar el individuo, es decir, no pueden garantizarle la areté en su acción.

Dice Maclntyre al respecto que las normas son enunciados generales que ponen límites a una actuación guiándola hacia el bien. Esa generalidad y esa limitación

12 "It is necessary, if life is to be meaningful, for us to be able to engage in long-term projects, and this requires predictability; it is necessary, if life is to be meaningful, for us to be in possession of ourselves and not merely to be the creations of other's projects, intentions and desires, and this requires unpredactibility". 
provocan que, a la hora de actuar, ninguna norma (o conjunto de normas) determine por sí misma cómo responder correctamente a una situación. Ello se debe a que las reglas que hay que respetar siempre (las referidas al bien del florecimiento, que son las de la ley natural) nunca bastan para determinar cómo hay que comportarse en la acción concreta, pues definen sólo parcialmente el tipo de bondad al que se aspira (el todo que es la felicidad, el florecimiento) (Maclntyre, 1990a: 139; 1992: 143; 1999: ix$\mathrm{x})$. Y, en el caso de las normas que dependen de las circunstancias - las de las prácticas principalmente-, es necesario juzgar su pertinencia o no (Maclntyre, 1988: 31-32).

El uso y la aplicación concretos de unas reglas no dependen ni están especificados por otras reglas sino que dependen de las cualidades del sujeto (de sus virtudes, y de la prudencia especialmente). De ahí que la excelencia -la areté en la acción- nunca se pueda reducir a mera aplicación de reglas (Maclntyre, 1988: 32). Por tanto, no existe ningún tipo de norma, ni prohibiciones inviolables ni prescripciones que puedan orientar la acción por sí mismas (Maclntyre, 1999: 94; también: 1998: 106).

Pienso que se puede decir que las virtudes operativizan, materializan las reglas. Para Tomás de Aquino, las reglas requieren las virtudes porque actuar según tales reglas sólo es bueno si es expresión de virtudes y porque el seguimiento de las reglas en sí mismo requiere la virtud de la prudencia (en Maclntyre, 1988: 193-194). Recoge esta idea Maclntyre al concluir que comportarse virtuosamente implica más que el mero 'uso' de normas (Maclntyre, 1999: 93-94).

No obstante, el calado de la virtud es mucho más hondo, pues no sólo influye en la aplicación de normas sino también en su definición. Es decir, las virtudes son necesarias también para establecer las normas (morales y también civiles). Aunque la prudencia tiene un papel eminente, tanto las virtudes morales como las intelectuales (y la prudencia tiene un carácter intelectual y moral al tiempo) son necesarias para una correcta definición de las normas (Maclntyre, 1988: 115-116). Son las virtudes, pues, las que deben guiar la definición de las reglas (Maclntyre, 1981: 119-120). 
Por todo ello, convengo con Daniel Innerarity cuando dice, refiriéndose a la virtud (y a la acción ética), que ésta "nada tiene que ver con la rutinaria seguridad de quien se preocupa sólo por los límites de lo permitido y busca un recetario o un código penal. (...) Pero la virtud auténtica es vida en plenitud. (...) La virtud no es algo que pueda aprenderse como se adquiere un conocimiento teórico o una destreza técnica. Si no fuera así, entonces saber qué es la felicidad equivaldría a ser feliz y conocer los preceptos de la poética sería lo mismo que ser un buen poeta. La lógica de la razón práctica es la de la 'inventio', es decir, descubrimiento e invención a un tiempo" (Innerarity, 1992: 42-43).

Me parece importante y sugerente la relación que establece Innerarity entre la razón práctica y la inventio. Pienso que la areté en la acción implica un proceso creativo. Por la variabilidad de circunstancias y por las características del agente (caracteriológicas, afectivas, etc.), realizar la mejor acción aquí y ahora supone siempre, hasta cierto punto, una novedad ética. En relación con las normas, dicha novedad radica en que, ante una situación determinada, el agente debe seleccionar qué norma o normas se relacionan con ella, proceso que no es automático. Y, a la vez, a la hora de aplicarlas, la vivencia y la manera de encarnar esas normas dependen totalmente de las características del sujeto. Se puede decir que el bien se hace, mientras que las normas se siguen. Hacer y realizar el bien implica seguir las normas, pero no es, como hemos visto, un seguimiento automático y estático, pues el proceso de realizar el bien, es decir, de hacerlo real aquí y ahora, tampoco lo es. Toda acción toda búsqueda de la areté en la acción- es una novedad.

\section{Conclusión}

Hemos visto cómo las reglas juegan un papel considerable pero complementario - si se puede hablar así- en la ética. Éstas deberían tener menor relevancia en la reflexión y en el debate moral. Si se me permite la expresión, la ética debería desnormativizarse. Actualmente, el debate en torno al establecimiento de 
normas aceptables universalmente (o comunitariamente) tiende a ocupar un lugar eminente en los debates y la reflexión ética. Ciertamente, las normas son importantes, pero hacerlas objeto principal de atención (separándolo de otros elementos del comportamiento) lleva, por una parte, a que los debates en torno a ellas queden inconclusos; $y$, por otra, a sociologizar la ética, dejando aparte aspectos más importantes (como, por ejemplo, el desarrollo armónico del carácter del individuo, o una explicación total y profunda de la acción humana).

Pienso que habría que recuperar o dar más importancia a la atención al individuo en la ética, tanto teórica como prácticamente (en parte, como contrapartida lógica de la desnormativización). La ética - si puede decirse así- debería subjetivizarse, en el sentido de situar su centro e interés en el sujeto. Desde el punto de vista teórico, la ética debería volver a intentar la pretensión de explicar no sólo la acción y sus elementos relacionados, sino también la vida (como un todo, como unidad) de la que una acción forma parte. Desde el punto de vista práctico, pienso que debería acentuarse el papel del sujeto en la explicación de las acciones y las reglas. Como saber práctico sobre lo contingente, la ética debería recuperar el papel del hombre -el hombre bueno- como referente. El bien es algo eminentemente personal (Aristóteles, Ética nicomáquea, l, 1095b, 25). Dice Aristóteles que el hombre bueno (virtuoso) es la medida de todas las cosas (Ética nicomáquea, IX, 1166a, 10-15). Es él el que, gracias a sus cualidades y su desarrollo moral, define lo realmente verdadero, valioso y placentero: "Ia medida de cada cosa es la virtud y el hombre bueno como tal" (Ética nicomáquea, X, 1176a, 15-20; 1176b, 25-30) $)^{13}$.

\section{Referéncias}

ARISTÓTELES (1998), Metafísica, trad. García Yebra, Gredos, Madrid, 3a reimpr.

- (1998) Ética nicomáquea, trad. Pallí Bonet, Gredos, Madrid, 4ạ reimpr.

INNERARITY, D. (1992), Libertad como pasión, Eunsa, Pamplona.

${ }^{13}$ En este sentido, convendría revitalizar el papel de los modelos en ética. 
MACINTYRE, A. (1971), Against the Self-Images of the Age: Essays on Ideology and Philosophy, Duckworth (y Trinity Press), London; y: Schocken Books, N. York, $x+284$ pp. - (1981), After Virtue: A Study in Moral Theory, Duckworth, London; y: University of Notre Dame Press, Notre Dame (Indiana), ix+252 pp.

- (1988), Whose Justice? Which Rationality?, Duckworth, London; y: University of Notre Dame Press, Notre Dame (Indiana), xi+410 pp.

- (1990a), Three Rival Versions of Moral Enquiry: Encyclopaedia, Genealogy, and Tradition, Notre Dame University Press, Notre Dame (Indiana); y: Duckwort, London, $\mathrm{x}+241 \mathrm{pp}$.

- (1990b), First Principles, Final Ends and Contemporary Philosophical Issues, Marquette University Press, Milwaukee.

- (1990c), "The Privatization of Good. An Inaugural Lecture", The Review of Politics, v. 52, n. 3 (Summer), pp. 344-361.

- (1991), "Nietzsche O Aristotele?", en Conversazioni Americane, Giovanna Borradori, (ed.), Editori Laterza, Roma-Bari, pp. 169-187. Trad. inglesa: “Nietzsche or Aristotle?", en (1994) The American Philosopher: Conversations with Quine, Davidson, Putnam, Nozick, Danto, Rorty, Cavell, Maclntyre, and Kuhn, G. Borradori, University of Chicago Press, Chicago, pp. 137-152. Reimpreso con el título "An Interview with Giovanna Borradori" en: (1998) The Maclntyre Reader, Kelvin Knight (ed.), Polity Press, Cambridge, pp. 255-66. Usamos esta reimpresión para este trabajo aunque indicamos en el texto el año de la publicación original.

- (1992), "Plain Persons and Moral Philosophy: Rules, Virtues and Goods", American Catholic Philosophical Quarterly, v. 66, n. 1 (Winter), pp. 3-20. Reimpresión (con algunos cambios de detalle): (1998) The MacIntyre Reader, Kelvin Knight (ed.), pp. 13652. En este trabajo, se citan las páginas de la reimpresión.

- (1994) "How Can We Learn What Veritatis Splendor Has To Teach?", The Thomist, v. 58, pp. 171-195. 
- (1996) "Wahre Selbsterkenntnis durch Verstehen unserer selbst aus der Perspektive anderer" (interview with Dmitri Nikulin), Deutsche Zeitschrift für Philosophie, v. 44, n. 4, pp. 671-683.

- (1998) "Aquinas's Critique of Education: Against His Own Age, Against Ours", en Philosophers on Education: New Historical Perspectives, A. Oksenberg Rorty (ed.) Routledge, London-N. York, pp. 95-108.

- (1999) Dependent Rational Animals: Why Human Beings Need the Virtues, Duckworth, London; y: Open Court Publishing, Chicago and La Salle (Illinois).

- (2000) "Theories of Natural Law in the Culture of Advanced Modernity", en Common Truths: New Perspectives on Natural Law, Edward B. McLean (ed.), ISI Books, Wilmington, Delaware, pp. 91-115.

- (2006a), Edith Stein: A Philosophical Prologue. Continuum, London.

- (2006b), Ethics and Politics. Selected essays, vol. 2, CUP, N. York.

- (2009) "Intractable Moral Disagreements" y "From Answer to Questions: A Response to the Responses", en CUNNINGHAM, L. (ed.), Intractable Disputes about the Natural Law. Alasdair Maclntyre and Critics, University of Notre Dame Press, Indiana, pp. 1-52 (cap. 1) y pp. 313-351 (cap. 10).

RUSSELL, Jack (2003), On MacIntyre, Thomson Learning (Wadsworth), Belmont. TOMÁS DE AQUINO (2001), Suma de teología, BAC, Madrid, 4a reimpr.

Doutor em Filosofia pela Universidad Internacional de Cataluña Professor de Ética, Bioética, Antropologia na University of Antonio de Nebrija/San Rafael-Nebrija Center E-mail: davidlorenzoes@yahoo.es 